\title{
Comparison of Phase Evolution and Sintering Properties of Ti-10Mo Alloys Prepared using Bulk Scrap and Blended Elemental (BE) Powder
}

\author{
In-Hyeok Choi ${ }^{1 \dagger}$, Jung-Min $\mathrm{Oh}^{2 \dagger}$, Daeyoung $\mathrm{Kim}^{3}$, Jong-Woong $\mathrm{Kim}^{4}$, and Hanjung Kwon ${ }^{4, *}$ \\ ${ }^{1}$ R\&D center, HANNAE For T Co., Ltd., Dangjin 31702, Republic of Korea \\ ${ }^{2} R \& D$ center, INNOMXENE Co., Ltd., Daejeon 34436, Republic of Korea \\ ${ }^{3}$ Advanced Geo-materials R\&D Department, Pohang Branch, Korea Institute of Geoscience and Mineral Resources, \\ Pohang 37559, Republic of Korea \\ ${ }^{4}$ Division of Advanced Materials Engineering and Research Center for Advanced Materials Development, \\ College of Engineering, Jeonbuk National University, Jeonju 54896, Republic of Korea
}

\begin{abstract}
In this study, Ti-10Mo alloy powder and sintered bodies were successfully fabricated using a threestep process of acid/organic solvent cleaning, hydrogenation/dehydrogenation (HDH) of bulk Ti-Mo scrap, and pressureless vacuum sintering of the alloy powder. This process can be used to recycle valuable Ti alloy. And unlike remelting-based recycling processes, in which elements vaporize, this process maintains the original ratio of Ti and Mo and results in no loss of elements during HDH and sintering. We note, however, that the phases in the resulting Ti-Mo alloy were changed by HDH and the sintering processes, and as a result the phases of the alloy evolved differently than those of alloys prepared by the blending of Ti and Mo powders. XRD results of the processed sintered bodies revealed different pathways for the transformation of the crystal structure. As sintering temperature increased, the dominant phase changes in the crystal structure of the sintered bodies that used commercial powders were $a^{\prime} \rightarrow a^{\prime}+a^{\prime \prime}$, while the phase changes for the recycled Ti-10Mo sintered bodies were $a^{\prime}+a^{\prime \prime} \rightarrow a^{\prime \prime}+B$. Although the density of the sintered body using commercial materials was generally higher at a lower sintering temperature than the density of the sintered body that used recycled powder, at $1673 \mathrm{~K}$ their respective densities were nearly identical. Moreover, the sintered body that contained scrap showed superior Vickers hardness after the dual phase $(a+b)$ of Ti was reached, despite its relatively low density.
\end{abstract}

(Received 13 September 2021; Accepted 16 November 2021)

Keywords: Ti-10Mo alloy, sintering, pre-alloyed, XRD, recycling, hydrogenation-dehydrogenation

\section{Introduction}

Titanium (Ti) and its alloys have received considerable attention in aerospace, mechanical engineering, and shipbuilding industries because of their excellent physical, thermal, and chemical properties [1-4]. Despite their practical use in various applications, however, they still play a relatively minor role in mass applications due to their high production costs. This makes it desirable to develop a lower cost process for $\mathrm{Ti}$ and its alloys. Conventional $\mathrm{Ti}$ alloy products have typically been prepared using thermo-chemical

\footnotetext{
These authors contributed equally to this work as the first author. - 최인혁 · 오정민 · 김대영: 연구원, 김종웅 · 권한중: 교수 *Corresponding Author: Hanjung Kwon

[Tel: +82-63-270-2299, Fax: +82-63-270-2305, E-mail: hjkwon@jbnu.ac.kr] Copyright (C) The Korean Institute of Metals and Materials
}

or mechanical processes such as casting, mechanical alloying, and other specific techniques [1-4]. However, these processes have drawbacks, including high processing costs, difficulty of machining, and materials loss. To address the problem of high costs associated with $\mathrm{Ti}$ and $\mathrm{Ti}$ alloys, numerous studies have been conducted and patents issued centering on scrap-recycling technologies [5-8]. Among these, one technique has gained increasing attention [9-11], which involves powdering metal scraps using methods such as hydrogenation-dehydrogenation (HDH), combined with a sintering process. The HDH process is well known to be the most economical method of fabricating $\mathrm{Ti}$ and $\mathrm{Ti}$ alloy powders [8]. Because Ti and Ti alloys have a high solubility in hydrogen, it is easy to form titanium hydrides by reacting the $\mathrm{Ti}$ and $\mathrm{Ti}$ alloys with hydrogen. In the HDH process, the 
formation of the hydride phase by hydrogen inclusion into the Ti lattice causes expansion of the lattice, and as a result, the $\mathrm{Ti}$ and $\mathrm{Ti}$ alloys are easily transformed to powder even by low impact crushing.

Problems such as stress shielding in human bones or the elution of aluminum and vanadium have been reported with Ti-6Al-4V, one of the most widely used titanium alloys in the medical field [12-16]. For this reason, there is considerable interest in alternative $\mathrm{Ti}$ alloys as potential substitutes. In particular, Ti-Mo alloys have received attention for use in biomedical applications because of their non-toxicity, excellent corrosion resistance, and biocompatibility with human bone [17]. It is known that adding 10 mass $\%$ Mo enhances various properties that increase their suitability for biomedical applications, due to the dual phases, $(\alpha+\beta)$ phases of Ti [18].

In this study, we prepared Ti-10Mo alloy powder and sintered bodies using Ti-Mo alloy scrap. To compare their respective structural and mechanical properties, we produced conventional Ti-Mo alloy by blending element powders with commercially pure (CP) Ti and Mo powders. As the sintering temperature changed, the different characteristics of the two types of sintered bodies were investigated by density measurement, X-Ray Diffraction, and the Vickers hardness test.

\section{Experimental Methods}

Figure 1 shows the flow chart of the experiment process. Ti-Mo alloy scrap was obtained by cutting scraps of consumable electrodes used in vacuum arc re-melting (VAR) [8]. The weight ratio of the $\mathrm{Ti}$ and Mo powder was 9:1 by mass \%. Before the HDH powdering process, we immersed the Ti-10Mo scrap in $1 \mathrm{~mol} / \mathrm{L}$ of $\mathrm{HCl}$ solution for $1 \mathrm{~min}$, followed by ultrasonic cleaning in ethanol for $1 \mathrm{~h}$. The cleaned alloy scrap was treated in a vacuum furnace for hydrogenation at a temperature of $873 \mathrm{~K} . \mathrm{H}_{2}$ gas $(6 \mathrm{~N})$ was introduced into the furnace to maintain pressure between $5 \times$ $10^{5}$ and $7 \times 10^{5} \mathrm{~Pa}$ for $2 \mathrm{~h}$. The hydrogenated Ti-10Mo scrap was pulverized and sieved by mechanical processing, and the sieved Ti-10Mo alloy powder was dehydrogenated under a vacuum of $6.7 \times 10^{-3} \mathrm{~Pa}$ at $973 \mathrm{~K}$ for $2 \mathrm{~h}$.

CP Ti and Mo powders (99.9\%, High Purity Chemicals,
(2)

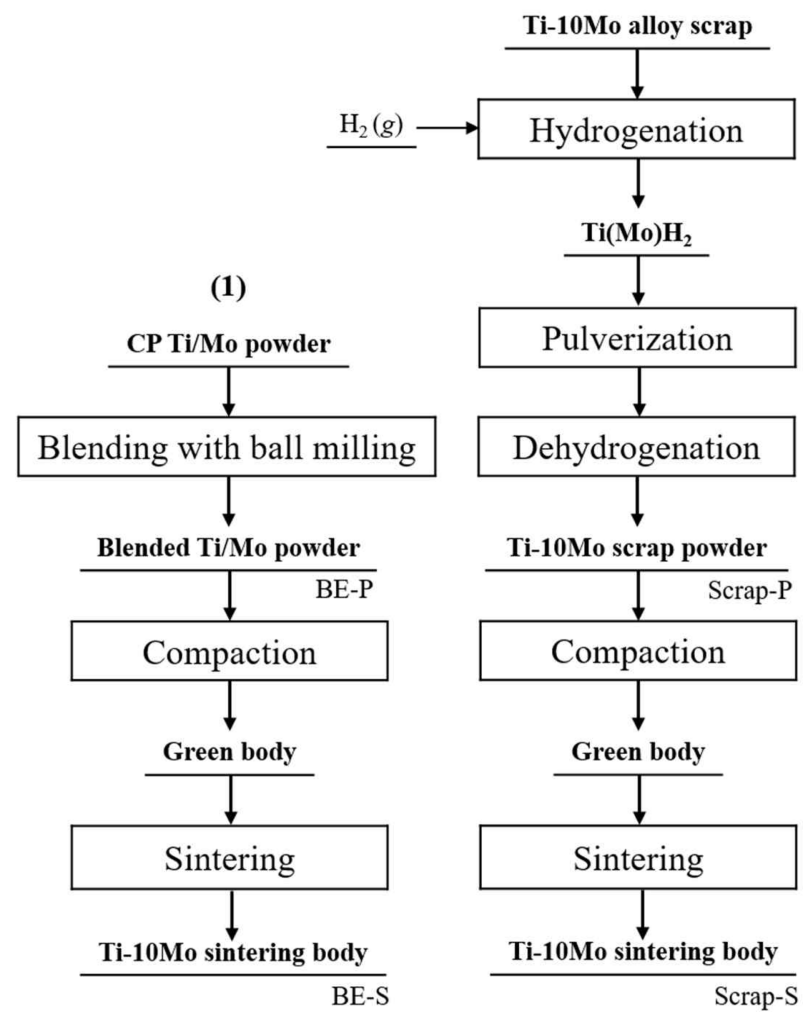

Fig. 1. Flow diagram of the experiment procedure, (1) Conventional method using commercial metal powder; (2) Recycling process using Ti-10Mo alloy scrap.

Japan) were used for the preparation of the conventional alloy metal. Both metal powders were blended with ethanol by ball milling for $24 \mathrm{~h}$ for uniform composition. The weight ratio of the steel ball to powder was 10:1. Ball milling was conducted with a rotation speed of $300 \mathrm{rpm}$. Both powders were compacted in a steel mold of $5 \mathrm{~mm}$ diameter and $3 \mathrm{~mm}$ height under a pressure of $2000 \mathrm{kgf} / \mathrm{cm}^{2}$ using a vertical press. Particle morphology was observed using scanning electron microscopy (SEM, JSM-6380LA, JEOL, Japan).

Sintering temperatures were in the range of $1273-1673 \mathrm{~K}$ under a vacuum of $1 \times 10^{-3} \mathrm{~Pa}$ for $2 \mathrm{~h}$ respectively. The chemical composition of the Ti-10Mo scrap used as raw materials was determined by glow discharge mass spectroscopy (GDMS, Autoconcept GD90, MSI Ltd., UK), and are listed in Table 1 [19]. The densities of the sintered bodies were measured by Archimedes method. X-Ray Diffraction (XRD) analysis (Rigaku, RTP 300 RC, Japan) 
Table 1. Chemical composition of Ti-10Mo scrap as raw materials.

\begin{tabular}{ccccccccc}
\hline $\begin{array}{c}\text { Ti-10Mo scrap } \\
\text { (mass\%) }\end{array}$ & $\mathrm{Fe}$ & $\mathrm{Mg}$ & $\mathrm{Al}$ & $\mathrm{Si}$ & $\mathrm{Cl}$ & $\mathrm{Ca}$ & $\mathrm{Ni}$ & $\mathrm{Cu}$ \\
\cline { 2 - 26 } & 0.021 & 0.011 & 0.028 & 0.028 & 0.064 & 0.012 & 0.013 & 0.003 \\
\hline
\end{tabular}

was conducted to investigate phase changes in the sintered bodies. The oxygen concentration of the powder and the sintered bodies of Ti-10Mo alloy was measured using a gas analyzer (LECO TCH-600, USA). The oxygen analyzer device used in this experiment had an average error range of $\pm 50 \mathrm{ppm}$. Hardness was tested with a $250 \mathrm{~g}$ load using a Vickers hardness tester (MKV-E, Akashi, Japan). The sintered bodies were etched using Kroll solution (2\% $\mathrm{HNO}_{3}+1 \% \mathrm{HF}$ ), and the cross-sectional structures of the specimens were observed by optical microscope (LEICA, DMC2900, Germany).

\section{Results and Discussion}

The two types of Ti-10Mo powder prepared by blending the CP Ti and Mo powders and by HDH using Ti-10Mo alloy bulk scrap, are hereafter referred to as BE-P (blended elemental powder) and SCRAP-P (pre-alloyed powder). The sintered bodies using BE-P and SCRAP-P are respectively referred to in the descriptions that follow as BE-S (sintered body using BE-P) and SCRAP-S (sintered body using SCRAP-P).

SEM images of the BE-P and SCRAP-P are shown in Fig. 2 displaying particle shape and size. The BE-P has a flat flake shape and an irregular size (Fig. 2(a)), while each particle of SCRAP-P has an angular spherical shape and a similar size distribution. The green densities of the BE-P and SCRAP-P were $70.2 \%$ and $65.1 \%$, respectively. The difference in green density between the two types of powder may be due to differences in particle shape and size distribution, since the uniformity of particle and pore sizes of the metal powders is one of the key factors affecting the sintered body or application [12].

We employed XRD analysis to analyze the transformation of the crystal structure during the powdering process by $\mathrm{HDH}$, and using the blended elemental method (Fig. 3). BEP (Fig. 3(a)) shows a mixed phase of $\alpha^{\prime}-\mathrm{Ti}$ and Mo without mechanical phase transformation from the ball milling process. Ti-10Mo bulk scrap was shown to be $\beta$-Ti phase
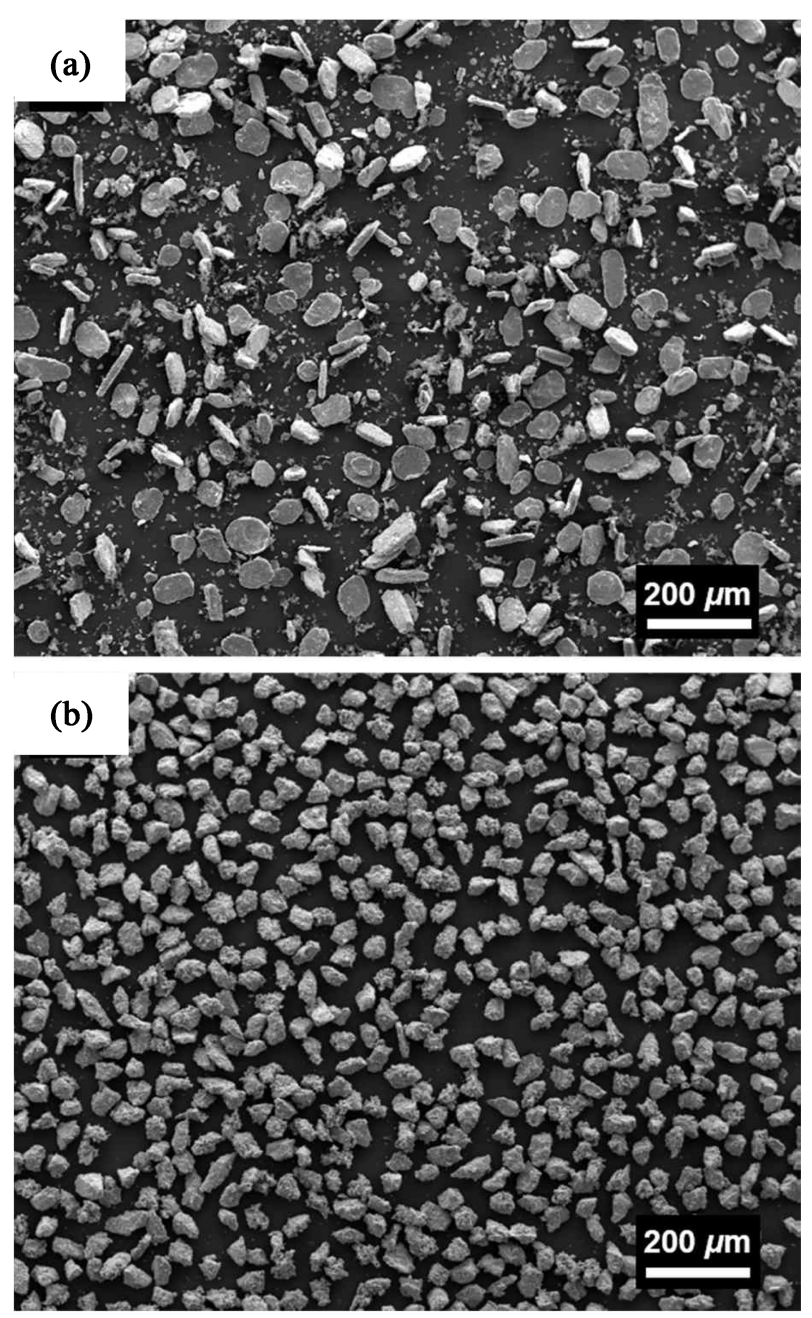

Fig. 2. SEM images of pulverized Ti-10Mo powder from commercial metal powder by ball milling, BE-P, (a); Ti-10Mo scrap by HDH process, SCRAP-P, (b).

with a partial $\omega$-Ti phase (Fig. 3(b)), confirming that the Mo in the feedstock was fully solubilized in the Ti lattice. XRD results for the hydrogenated Ti-10Mo bulk scrap resulted in the $\mathrm{TiH}_{2}$ phase, as shown in Fig. 3(c), indicating that hydrogen atoms were completely diffused in the Ti matrix under the given hydrogenation conditions.

Figure 3(d) shows that dehydrogenated SCRAP-P has a $\alpha^{\prime}$ (hexagonal), and $\alpha^{\prime \prime}$ (orthorhombic, high solid solution degree of Mo) $+\beta$-Ti (near $\beta$ ) as the main phase. According to the binary phase diagram of $\mathrm{Ti}$ and Mo, the Ti-10Mo alloy is a 


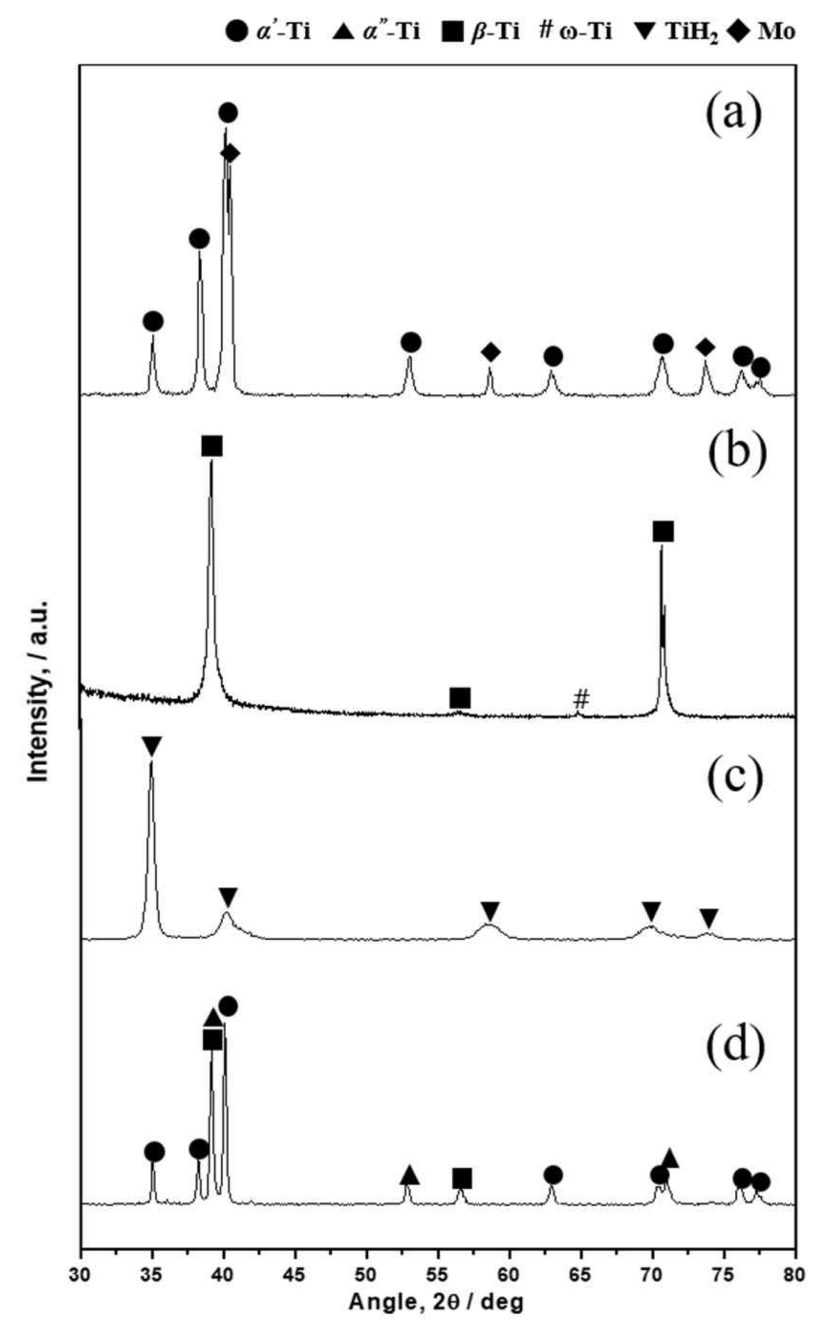

Fig. 3. XRD patterns of blended elemental powder $(\mathrm{CP} \mathrm{Ti}+10$ mass\% Mo) (a); Ti-10Mo alloy scrap (b); Ti-10Mo alloy scrap powder (hydrogenated (c); dehydrogenated (d)).

homogeneous solid solution [20, 21]. When 10 mass $\%$ of Mo is alloyed to titanium, the $\beta$ titanium phase is present $[8,22]$. In the XRD result, the phase of the Ti-10Mo alloy scrap is observed to be $\beta$ phase. However, after the HDH process the homogeneous solid solution of Ti-10Mo alloy becomes $\alpha$ and $\beta$ titanium phases. This phenomenon has also been reported in other homogeneous solid solutions of Ti-V alloy [8]. Comparing the crystal structure of the raw scrap ( $\beta$-Ti) with the dehydrogenated Ti-10Mo scrap powder, the Mo in the Ti lattice becomes partially segregated during the $\mathrm{HDH}$ process because the appearance of the $\alpha$ phase in the Ti-Mo alloy is closely related to the solid solution degree of Mo [22].

Figure 4 shows the XRD results of the sintered bodies with various sintering temperatures, used to investigate phase transformations in the BE-S and SCRAP-S, and the different phase-change behavior of the two sintered bodies. For the BE-S in Fig. 4(a)-(e), the main phase transformation was from $\alpha^{\prime}$ to $\alpha^{\prime \prime}$ phase. Ho et al. and Bagariaskii et al. have reported that the shifting of XRD peaks from $\alpha^{\prime}$-Ti is a direct indication of orthorhombic $\alpha^{\prime \prime}-\mathrm{Ti}$, depending on the solid solution degree of Mo [22-23]. The observed phase transformation from $\alpha^{\prime}-\mathrm{Ti}(10 \overline{1} 1)$ to $\alpha^{\prime \prime}-\mathrm{Ti}(111)$ for the BE$\mathrm{S}$ in Fig. 4 is in line with the existing literature [22]. In addition, XRD peaks of the free Mo metal phase were found at $1273 \mathrm{~K}$ at around $41^{\circ}(110), 58^{\circ}(200)$, and $73^{\circ}$ (211). However, these Mo peaks disappeared above $1373 \mathrm{~K}$ due to the solid solution in the Ti matrix. Accompanying the disappearance of the free Mo peaks, $\alpha$ "-Ti peaks (111) developed at $1473 \mathrm{~K}$, and grew steadily as a function of sintering temperature, rather than the appearance of $\beta$-Ti. This result indicates that the phase transformation of $\alpha^{\prime}-\mathrm{Ti}$ (low Mo solute content) did not directly transition to the $\beta$ Ti phase. Since BE-S was produced from a simple mixture of $\mathrm{Ti}$ and Mo metal powders, the equilibrium structure of the blended composition of the Ti-10Mo binary system required more thermal activation than its scrap (pre-alloyed) to attain the complete diffusion of Mo.

Figure 4(f)-(j) shows the XRD results for SCRAP-S in relation to sintering temperature. The phase transformation in SCRAP-S is also similar to that of BE-S ( $\left.\alpha^{\prime}-\mathrm{Ti} \rightarrow \alpha^{\prime \prime}-\mathrm{Ti}\right)$. Also, it was found that $\beta$-Ti becomes stable as the sintering temperature increases in both cases. This is because the stability of the $\beta$-Ti is higher than that of $\alpha$-Ti above $1000 \mathrm{~K}$, regardless of the composition of the Ti-Mo system [24]. However, the dominant change in crystal structure was from $\alpha "+\beta$ Ti to $\beta$-Ti. The predominant phases at $1273 \mathrm{~K}$ were $\alpha "-$ Ti and $\alpha^{\prime \prime}+\beta$ Ti with sub $\alpha^{\prime}$-Ti peaks in Fig. 4(f), although the $\alpha^{\prime \prime}+\beta$ Ti phase was close to $\beta$-Ti as the sintering temperature increased. This indicates that the diffusion of Mo into the Ti lattice continued to occur via thermal activation.

In addition, most of the sub $\alpha^{\prime}-$ Ti peaks, such as $(10 \overline{1} 2)$, $(11 \overline{2} 0)$, and $(11 \overline{2} 2)$ in Fig. 4, transformed into $\alpha "-T i$ simultaneously, while $\alpha^{\prime \prime}-\mathrm{Ti}$ at $1673 \mathrm{~K}$ dominated the crystal structure $\alpha\left(\alpha^{\prime}\right.$ and $\left.\alpha^{\prime \prime}\right)$ Ti. Accompanying the almost complete phase transformation from $\alpha^{\prime}$ into $\alpha^{\prime \prime}-\mathrm{Ti}, \beta-\mathrm{Ti}$ consequently appeared as a main phase. From the XRD 

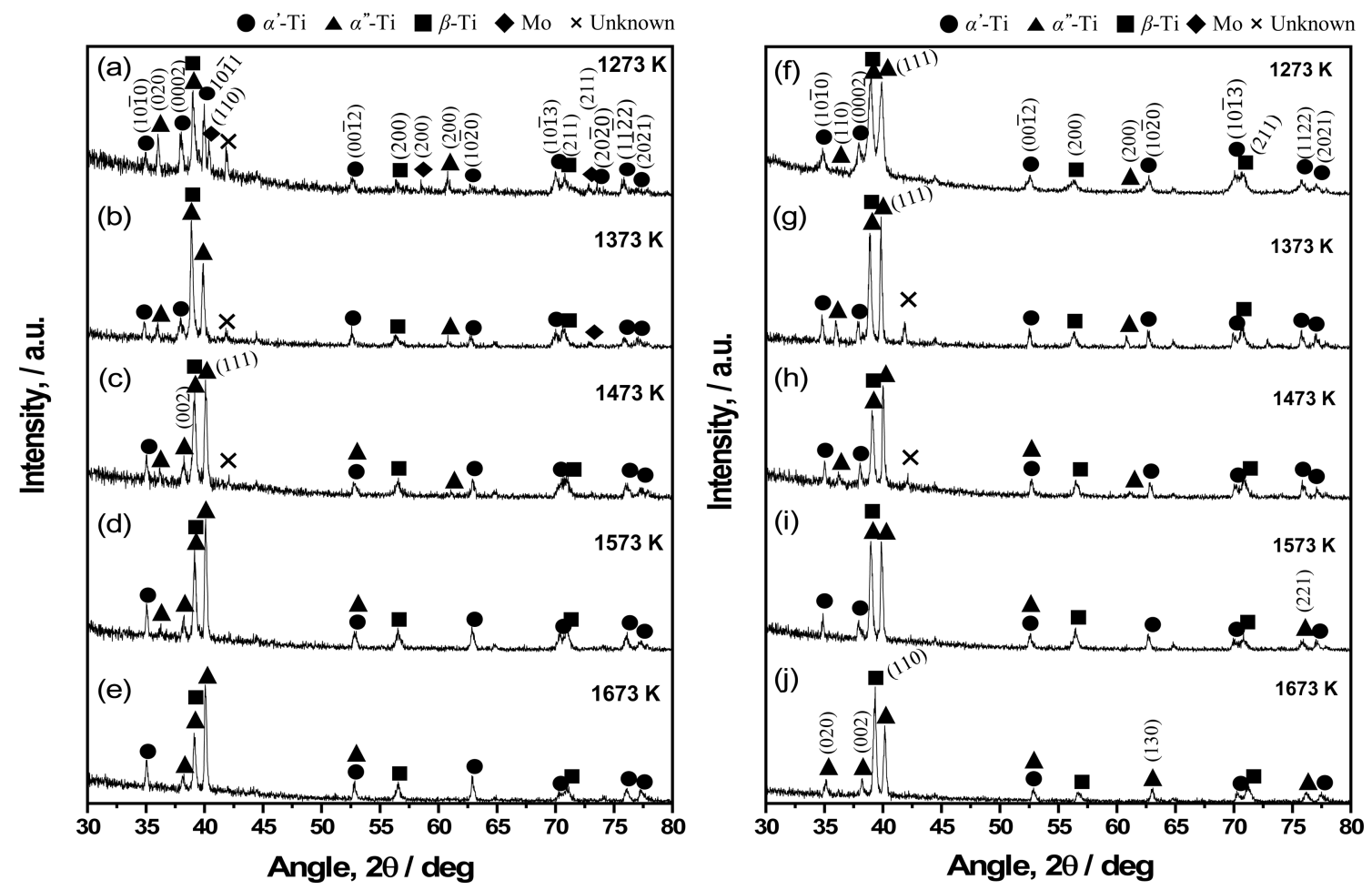

Fig. 4. XRD patterns of BE-S ((a) (e)) and SCRAP-S ((f) (j)) as a function of sintering temperature.

Table 2. Variation of oxygen concentrations of the powder and sintered bodies of Ti-10Mo alloy.

\begin{tabular}{ccccccc}
\hline & Powder & \multicolumn{5}{c}{ Sintered bodies (mass\%) } \\
\cline { 3 - 7 } & $\left(\begin{array}{ccccc}\text { mass\%) } \\
\right.$\cline { 3 - 7 }\end{array} & $1273 \mathrm{~K}$ & $1373 \mathrm{~K}$ & $1473 \mathrm{~K}$ & $1573 \mathrm{~K}$ & $1673 \mathrm{~K}$ \\
\hline BE-S & 0.425 & 0.346 & 0.349 & 0.404 & 0.402 & 0.406 \\
\hline SCRAP-S & 0.434 & 0.351 & 0.353 & 0.406 & 0.405 & 0.409 \\
\hline
\end{tabular}

spectra of the sintered bodies, SCRAP-S was able to attain the dual phase of Ti with less thermal activation than BE-S, presumably due to the different solid solution degree of Mo compared to the raw materials. Therefore, the recycling of Ti-10Mo alloy scrap using the powder metallurgical route may be worth pursuing as a means of reducing processing costs and energy. This is because the $\beta$-stabilization of $\mathrm{Ti}$ with 10 mass $\%$ Mo during the sintering process with SCRAP-P requires less time and thermal energy than BE-P under a given sintering condition. It may thus be more advantageous to use Ti-10Mo alloy scrap than blended powder due to the difficulty of achieving compositional homogeneity.

Oxygen has a strong influence on the physical properties of both the powder and bulk titanium; the higher the oxygen concentration, the lower the alloy density after the sintering process $[1,25]$. Table 2 lists oxygen concentrations for the Ti-10Mo alloy powder and sintered bodies. These were measured to be 0.425 and 0.434 mass $\%$, respectively, for the BE-P and SCRAP-P. After the sintering process, the oxygen concentrations of the BE-S specimens were shown to be $0.346,0.349,0.404,0.402$, and 0.406 mass $\%$ at sintering temperatures of 1273 - $1673 \mathrm{~K}$, respectively, while those of the SCRAP-S specimens were shown to be $0.351,0.353$, 0.406, 0.405, and 0.409 mass $\%$, respectively.

The oxygen concentrations for the BE-S and SCRAP-S increased slightly with increasing sintering temperature, possibly due to a reaction with the small amount of oxygen remaining in the vacuum chamber as the temperature increased. However, the deviation in oxygen concentration between BE-S and SCRAP-S was as low as $0.002-0.005$ mass $\%$. Therefore, the differences in the oxygen 

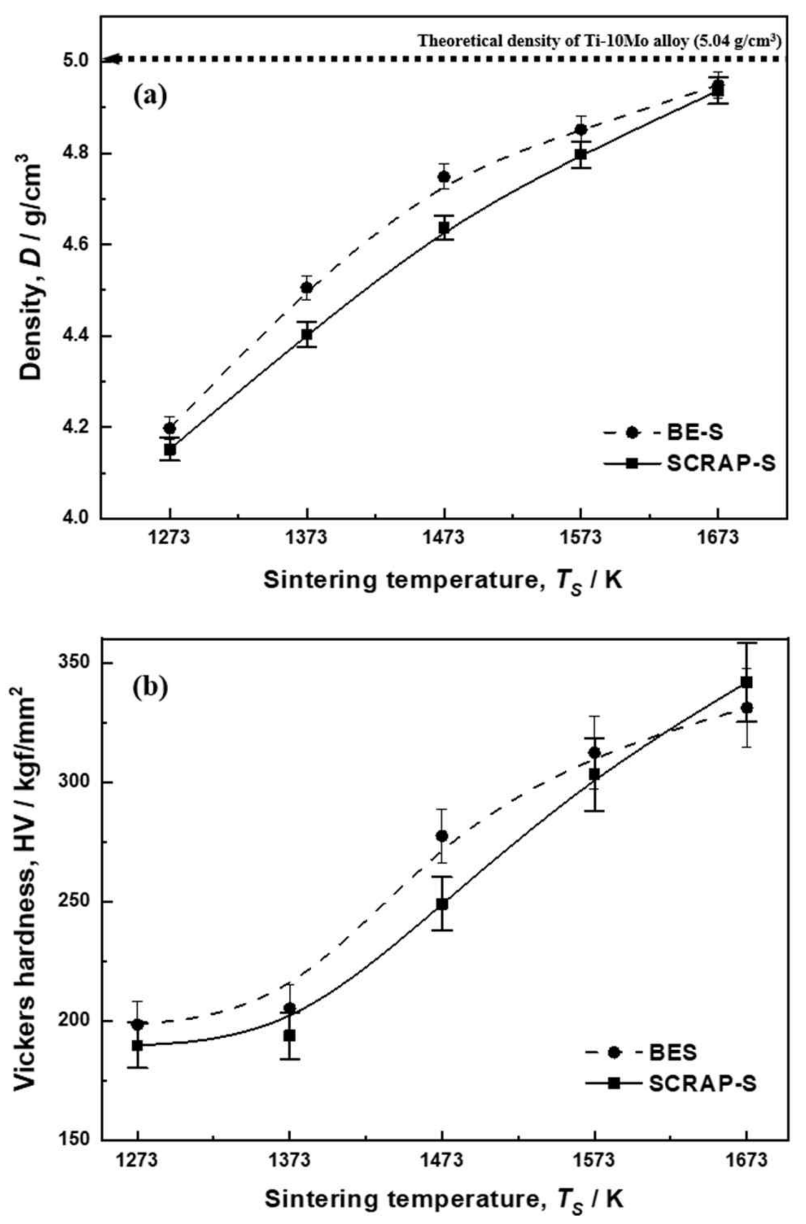

Fig. 5. Variation of density (a) and Vickers hardness (b) of BE-S and SCRAP-S as a function of sintering temperature.

concentrations of the BE-S and SCRAP-S were considered to have a negligible effect on the physical properties of the sintered bodies.

Figure 5 shows changes in the density (a) and Vickers hardness (b) of the BE-S and SCRAP-S as a function of sintering temperature. For each of the sintered bodies, the tendencies in both the density and Vickers hardness were consistent with the general densification of sintered bodies with increasing temperature. The density of the BE-S sintered bodies was slightly higher than that of SCRAP-S within the temperature range of $1273-1573 \mathrm{~K}$. However, at $1673 \mathrm{~K}$ the density of SCRAP-S was almost identical to that of BE-S. Maximum densification, achieved at $1673 \mathrm{~K}$, was 98\% for BE-S and $97.9 \%$ for SCRAP-S, compared to the theoretical density of the Ti-10Mo casting alloy $\left(5.04 \mathrm{~g} / \mathrm{cm}^{3}\right)$. The differences in density in relation to sintering temperature
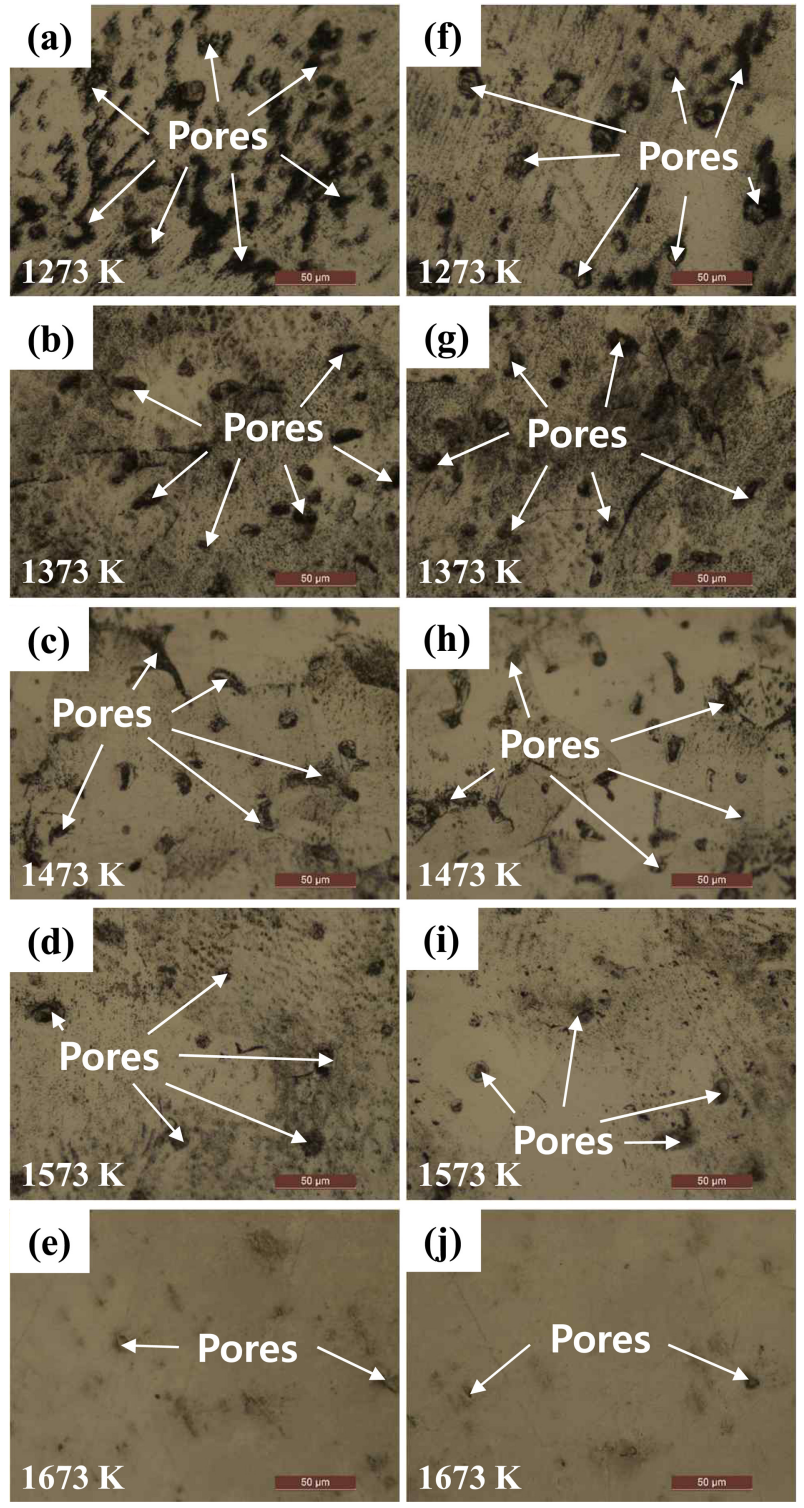

Fig. 6. Surface morphologies of BE-S ((a) (e)) and SCRAP-S $((\mathrm{f}) \sim(\mathrm{j}))$ as a function of sintering temperature.

for the two sintered bodies may be regarded as the difference in green density originating from their different particle morphologies.

In addition, Fig. 6 shows changes in the surface and crosssectional microstructures of the BE-S and SCRAP-S as a function of sintering temperature. The numbers of pores in BE-S and SCRAP-S decreased with increasing temperature. Both samples showed similar trends, which tended to be consistent with the density results.

As shown in Fig. 5(b), the Vickers hardness results 
corresponded to the results in Fig. 5(a). The values for SCRAP-S were similar to those for BE-S between 1273 and $1573 \mathrm{~K}$, in spite of their lower green density; however, they exceeded the value of BE-S at $1673 \mathrm{~K}$. This result is presumably due to solid solution strengthening by the different solute content in Ti [20]. The Vickers hardness of SCRAP-S in its dual phase was comparable to that of the Ti10Mo casting alloy [22].

It can thus be concluded that recycling Ti-10Mo alloy scrap using the powder metallurgical route is a more favorable approach, involving lower costs and higher efficiency than the conventional blending powder method.

\section{Conclusions}

In this study, we compared the advantages of producing Ti-10Mo powder and its sintered body from recycled Ti10Mo bulk scrap, compared with commercial powder. A superior Ti-10Mo alloy powder with $\alpha\left(\alpha^{\prime}+\alpha^{\prime \prime}\right)+\beta$ phase was obtained from the bulk scrap via the HDH process, compared to the commercial powder. According to the XRD results from the sintered bodies, the dominant crystal structure changes in BE-S and SCRAP-S were $\alpha>\alpha$ " and $\alpha '+\alpha " \rightarrow \alpha "+\beta$, respectively. Under identical sintering conditions, a dual phase was more easily attained in SCRAP-S than BE-S. While the density of the BE-S was higher than that of SCRAP-S between 1273 and $1573 \mathrm{~K}$, the difference between them diminished as temperature increased, and at $1673 \mathrm{~K}$, they became almost identical. The results of the Vickers hardness test were consistent the relationship between density and mechanical properties. Moreover, the increase in Vickers hardness for SCRAP-S was more pronounced than for BE-S. Eventually, the Vickers hardness of SCRAP-S, which had reached dual phase, became identical to or greater than that of BE-S at $1673 \mathrm{~K}$, and became comparable to that of cast Ti-10Mo alloy.

\section{Acknowledgements}

This work was partly supported by Korea Institute of Energy Technology Evaluation and Planning (KETEP) grant funded by the Korea government (MOTIE) (20217510100020). And this paper was proofread by the Writing Center at Jeonbuk National University.

\section{REFERENCES}

1. G. Lutjering and J. C. Williams, Titanium, Springer, Berlin Heidelberg (2003).

2. C. Leyens and M. Peters, Titanium and Titanium Alloys, Wiley-VCH Verlag GmbH \& Co. KGaA, Weinheim (2003).

3. H-J. Jeong, V. T. Luu, Y.-H. Jeong, S.-T. Hong, and H. N. Han, Korean J. Met. Mater. 58, 162 (2020).

4. J.-M. Oh, J. Yang, J. Yoon, and J.-W. Lim, Korean J. Met. Mater. 59, 149 (2021).

5. Y. Hatta, T. Sakai, T. Shiraki, T. Sannoeh, and O. Tada, Process for production of titanium alloy, United States Patent US 10/455,385 (2003).

6. B. M. Moon, J. H. Seo, H. J. Lee, K. H. Jung, J. H. Park, and H. D. Jung, J. Alloys Compd. 727, 931 (2017).

7. Y. G. Liu and M. Q. Li, J. Alloys Comp. 773, 860 (2019).

8. J. M. Oh, K. M. Roh, B. K. Lee, C. Y. Suh, W. Kim, H. Kwon, and J. W. Lim, J. Alloys Comp. 593, 61 (2014).

9. D. A. Small, G. R. Mackay, and R. A. Dunlap, J. Alloys Compd. 284, 312 (1999).

10. C. R. F. Azevedo, D. Rodrigues, and F. B. Neto, J. Alloys Compd. 353, 217 (2003).

11. F. H. Froes, D. Eylon, G. E. Eichelman, and H. M. Burte, J. Metals 32, 47 (1980).

12. M. Qian and F. H. Froes, Titanium powder Metallurgy: Science, Technology and Applications, ButterworthHeinemann, Oxford (2015).

13. C. E. Wen, M. Mabuchi, Y. Yamada, K. Shimojima, Y. Chino, and T. Asahina, Scripta Mater. 45, 1147 (2001).

14. D. M. Brunette, P. Tengvall, M. Textor, and P. Thomsen, Titanium in Medicine: Material Science, Surface Science, Engineering, Biological Responses and Medical Applications, Springer, Berlin Heidelberg (2001).

15. W. Geurtsen, Crit. Rev. Oral Biol. Med. 13, 71 (2002).

16. S. Lugowski, D. Smith, A. McHugh, and V. Loon, J. Biomed. Mater. Res. 25, 1443 (1991).

17. Z. Gao, Q. Li, F. He, Y. Huang, and Y. Wan, Mater. Des. 42, 13 (2012).

18. G. Rondelli and B. Vicentini, Biomaterials 23, 639 (2002).

19. J. M. Oh, B. K. Lee, C. Y. Suh, and J. W. Lim, J. Alloys Comp. 574, 1 (2013). 
20. H. Okamoto, Desk Handbook: Phase Diagram for Binary, ASM International (2000).

21. Z. Tarzimoghadam, S. Sandlöbes, K. G. Pradeep, and D. Raabe, Acta Mater. 97, 291 (2015).

22. W. F. Ho, C. P. Ju, J. H. and C. Lin, Biomaterials, 20, 2115 (1999).
23. I. A. Bagariaskii, G. I. Nosova, and T. V. Tagunova, Sov. Phys. Dokl. 3, 1014 (1959).

24. J. L. Murray, J. Phase Equilib. 2, 185 (1981).

25. J. M. Oh, I. H. Choi, C. Y. Suh, H. Kwon, J. W. Lim, and K. M. Roh, Met. Mater. Int. 22, 488 (2016). 